\title{
UM MODELO MULTICRITÉRIO CONSTRUTIVISTA PARA APOIAR A GESTÃO DA ATIVIDADE DE PESQUISA DA UNIVERSIDADE DE MINDELO
}

\section{A CONSTRUCTIVIST MULTI-CRITERIA MODEL TO SUPPORT THE RESEARCH MANAGEMENT OF THE UNIVERSITY OF MINDELO}

\author{
Thuine Lopes Cardoso \\ Formação em Ciências Contábeis pela Universidade Federal de Santa Catarina - UFSC, Santa \\ Catarina (Brasil). E-mail: thuinecardoso@ hotmail.com
}

\section{Sandra Rolim Ensslin}

Doutorado em Engenharia de Produção pela Universidade Federal de Santa Catarina - UFSC, (Brasil). E-mail: sensslin@gmail.com

\section{Ademar Dutra}

Doutorado em Engenharia de Produção pela Universidade Federal de Santa Catarina, UFSC, Brasil. E-mail: ademar.unisul@gmail.com

\section{Juscelino Dias}

Doutorado em Informática de Decisão e Doutorado em Engenharia e Gestão Industrial. Pesquisador da Universidade do Mindelo (Cabo Verde). E-email: judiascv@gmail.com 



\title{
UM MODELO MULTICRITÉRIO CONSTRUTIVISTA PARA APOIAR A GESTÃO DA ATIVIDADE DE PESQUISA DA UNIVERSIDADE DE MINDELO.
}

\section{RESUMO}

Este estudo tem como objetivo estruturar um modelo de avaliação de desempenho para apoiar a gestão da atividade de pesquisa da Universidade de Mindelo (UM), sediada em Cabo Verde, na África. Para tal, utilizou-se, como instrumento de intervenção, a Metodologia Multicritério de Apoio à Decisão-Construtivista (MCDA-C). A aplicação dessa metodologia permitiu a geração de conhecimento no pró-reitor de pesquisa da UM para identificação dos aspectos relevantes, necessários e suficientes, segundo a sua percepção, para avaliar e gerenciar o contexto. Entre os principais resultados, está a identificação: 1) de cinco áreas de preocupação: Capacitação; Estruturação da Pesquisa; Captação de Recursos; Operacionalização da Pesquisa; e, Resultados da Pesquisa; 2) de indicadores e do perfil de desempenho da UM; e 3) de indicadores com desempenho comprometedor, dentre eles: Percentual de participação docente em eventos científicos no último ano; Percentual de docentes com publicação em revistas de alto fator de impacto nos últimos dois anos; Percentual do corpo docente com doutorado; e, Percentual de pesquisas voltadas a temas ligados a problemas ambientais no último ano. Para esses indicadores, ações de melhorias foram sugeridas para alavancar o desempenho da atividade de pesquisa da UM. Nesse contexto, os autores deste estudo acreditam que a construção de um modelo personalizado para avaliar e gerir a atividade de pesquisa contribuirá para o desenvolvimento da pesquisa científica e para a geração de conhecimento proporcionada por ela.

Palavras-chave: Gestão Universitária. Pesquisa. Modelo Construtivista.

\section{A CONSTRUCTIVIST MULTI-CRITERIA MODEL TO SUPPORT THE RESEARCH MANAGEMENT OF THE UNIVERSITY OF MINDELO}

\begin{abstract}
This study has the purpose of structuring a performance evaluation model to support the research management of the University of Mindelo (UM), based in Cabo Verde, Africa. To this end, the instrument of intervention used was the methodology known as Multi-Criteria Decision Aiding - Constructivist (MCDA-C). The use of this methodology enabled the UM Research Pro-rector develop knowledge, which identifies the relevant, necessary and sufficient aspects, according to his perception, to evaluate and manage the university research management context. Among the main results achieved, the identification of the following is noted: 1) five areas of concern: Training; Research Structuring; Fundraising; Research Operationalization; and; Research Results; 2) performance profile and UM indicators; and 3) indicators with compromising performance, among them: a percentage of Professors taking part in scientific events over the last year; percentage of Professors with papers published in high-impact factor journals over the last two years; a percentage of Professors holding a doctoral degree; and a percentage of research related to environmental issues over the last
\end{abstract}


year. For these indicators, actions of improvement were recommended in order to improve UM research performance. In this context, the authors of this study believe that creating a specific model to evaluate and manage research will contribute to the development of scientific research and to the development of knowledge in the UM context.

Keywords: University Management. Research. Constructivist Model.

\section{INTRODUÇÃO}

A necessidade da avaliação de desempenho no ensino superior é impulsionada pela crescente concorrência entre as instituições, pelos cortes nas despesas públicas no financiamento das universidades por parte dos governos, pelo desejo de melhoria interna no desempenho e, também, pelas pressões das partes interessadas (Asif \& Searcy, 2014). Entre as pressões externas, exercidas pelas partes interessadas, estão os rankings que fornecem tabelas classificatórias com o desempenho das universidades na tentativa de fornecer informações para os usuários tais como: o público em geral, os políticos que financiam o sistema e potenciais alunos. Como no contexto externo, internamente, os gestores universitários são pressionados no sentido de direcionarem suas ações para o alcance dos objetivos estratégicos e para o aperfeiçoamento do desempenho institucional (Azma, 2010). De certa forma, as avaliações externas, possuem caráter compulsório; e, as internas, possuem caráter voluntário, normalmente destinadas à autoavaliação com o objetivo de comunicar à comunidade universitária sua contribuição para a melhoria do desempenho institucional, bem como promover maior consciência das atividades que estão sendo realizadas e das que não estão sendo realizadas (Azma, 2010).

Agyemang e Broadbent (2015) argumentam que a avaliação de desempenho das universidades deve considerar tanto o contexto interno, quanto o externo à própria organização, uma vez que há a necessidade de responder aos controles externos e ao ambiente regulatório, ao mesmo tempo que é importante considerar os objetivos que a organização pretende alcançar considerando todos os envolvidos. Entretanto, essa atividade avaliativa é complexa, uma vez que envolve vários stakeholders com objetivos e interesses distintos, como agências de financiamento, governo, potenciais alunos e o próprio corpo docente e pessoal técnico-administrativo da universidade (Colem, Joyce, Hassall \& Broadbent, 2003). Adicionalmente à dificuldade de envolver e/ou selecionar os stakeholders tem-se a questão da identificação/construção dos fatores-chave e indicadores que farão parte do processo avaliativo (Luneta, 2015; Assim \& Dercy, 2014; Azma, 2010). A literatura aponta que, os indicadores, além de fornecer mecanismo para monitorar e analisar o desempenho, também são utilizados para atender exigências legais, de transparência e de responsabilidade pública, fornecer informações para as várias partes interessadas, fornecer orientações sobre como proceder para a melhoria contínua durante a operação com recursos limitados e gerenciar os processos-chave da universidade (Asif \& Searcy, 2014).

Entre os processos-chave da universidade, está a pesquisa, haja vista que, as IES têm tradicionalmente definido, entre seus objetivos principais, a construção e a transmissão de conhecimento (Higgins, 1989), sendo esse, justamente, o propósito da pesquisa científica (Agyemang \& Broadbent, 2015). Assim, é necessário que o desempenho dos processos e da atividade de pesquisa dentro das universidades seja avaliado, para que o processo de criação e desenvolvimento de conhecimento possa ser aperfeiçoado e contribua, cada vez mais, para a comunidade científica e para a própria universidade (Agyemang \& Broadbent, 2015). 
Somado a isso, o governo de Cabo Verde reconheceu a necessidade da avaliação de desempenho dos processos-chave das universidades e determinou a avaliação institucional educacional, onde todas as IES passaram a ser sujeitas à avaliação de desempenho externa (Cardoso, 2014). Desse modo, tornou-se necessário o desenvolvimento de sistemas que possibilitem a avaliação de desempenho interna e possam subsidiar as IES cabo-verdianas na avaliação externa. Neste contexto, resgata-se a proposta de Ensslin, Ensslin, Back e Lacerda (2013, p.739) que passa a constituir-se como a filiação teórica que informa os autores da presente pesquisa, onde a avaliação de desempenho interna configura-se como um:

[...] processo para construir conhecimento no decisor, a respeito do contexto específico que se propõe avaliar, a partir da percepção do próprio decisor por meio de atividades que identificam, organizam, mensuram, ordinalmente e cardinalmente, integram os aspectos considerados como necessários e suficientes para sua gestão, permitindo visualizar o impacto das consequências das ações e seu gerenciamento (tradução nossa).

Assim, este estudo apresenta a seguinte pergunta de pesquisa: Quais aspectos devem ser considerados na avaliação da atividade de pesquisa de uma universidade, que apoiem a tomada de decisão no aperfeiçoamento do desempenho institucional? Para responder a essa pergunta, este estudo apresenta este objetivo geral: estruturar um modelo de avaliação de desempenho multicritério construtivista para apoiar a gestão da atividade de pesquisa da Universidade de Mindelo (UM), sediada em Cabo Verde, na África.

Para atender ao objetivo, a Metodologia Multicritério de Apoio à Decisão-Construtivista (MCDA-C) foi selecionada, como instrumento de intervenção, por sua perspectiva construtivista que permitirá ao gestor, aqui o pró-reitor de pesquisa da Universidade de Mindelo, identificar quais aspectos são necessários e suficientes para avaliar o desempenho da atividade de pesquisa da universidade, compreender os impactos das decisões tomadas e identificar ações de aperfeiçoamento.

Ressalta-se que, nenhum estudo utilizando a Metodologia MCDA-C foi encontrado na literatura consultada. As ferramentas encontradas na literatura para construção/estruturação de um modelo de avaliação de desempenho foram: Analytic Hierarchy Process (AHP) e Data Envelopment Analysis (DEA) (Asif \& Searcy, 2014; Bei \& Dongsheng, 2014; Korhonen, Tainio \& Wallenius, 2001). Nenhuma dessas ferramentas carece do viés construtivista, abordado na Metodologia MCDA-C, visando à construção de conhecimento no decisor a respeito do problema e do contexto vivenciado por ele.

Este estudo justifica-se quanto à importância, originalidade e viabilidade (Castro, 1977). É importante pela necessidade de gerenciar os recursos tangíveis e intangíveis relacionados à atividade de pesquisa universitária e, em particular, sobre como desenvolver indicadores de desempenho que reflitam o contexto único de uma instituição de ensino superior (Asif \& Searcy, 2014). Adicionalmente, a importância desta pesquisa pode também ser argumentada com base na visão de Korhonen, Tainio e Wallenius (2001) que ressaltam o fato de, apesar da centralidade da pesquisa em universidades, parece muito difícil avaliá-la e esse é um problema universal. É original por não terem sido encontrados, na literatura consultada, trabalhos que construam um modelo multicritério construtivista para a gestão da atividade de pesquisa. É viável pelo interesse do pró-reitor de pesquisa da Universidade de Mindelo na construção de um modelo personalizado que lhe forneça embasamento para as tomadas de decisões de forma transparente. 


\section{REFERENCIAL TEÓRICO}

\subsection{Avaliação de Desempenho}

A literatura sobre "Avaliação de Desempenho" está dividida em dois períodos. Do final da década de 1880 até a década de 1980, a literatura enfatizava apenas medidas financeiras e tinha como objetivo a gestão da produtividade em decorrência do aumento da industrialização (Ghalayini \& Noble, 1996; Bititci, Garengo, Dörfler \& Nudurupati, 2012). No final da década de 1980, com o surgimento de contextos organizacionais complexos, apenas medidas financeiras passaram a não atender as necessidades das organizações. Assim, as estratégicas foram alteradas e medidas não financeiras passaram a ser incorporadas no processo de avaliação de desempenho (Ghalayini \& Noble, 1996; Bititci et al., 2012). Dessa forma, as organizações passaram a desenvolver sistemas de mensuração e gestão de desempenho considerando medidas financeiras e não financeiras relacionadas aos objetivos e à estratégia organizacional para apoiar a gestão (Ghalayini \& Noble, 1996; Franco-Santos, Lucianetti \& Bourne, 2012).

O sistema de mensuração do desempenho aborda a fase de definição das métricas e as fases de coleta e interpretação de dados (Melnyk et al. 2014). Já o sistema de gestão compara os resultados reais com os esperados, busca compreender a razão das diferenças e irregularidades; e procura introduzir medidas corretivas para aperfeiçoar e melhorar o desempenho institucional. Esses dois sistemas devem ser utilizados de forma complementar para apoiar a decisão e juntos formam o "sistema integrado" de avaliação de desempenho organizacional (Melnyk et al., 2014, p. 175).

De acordo com Roy (1993) e Dias e Tsoukiàs (2004), a abordagem construtivista é a mais apropriada quando o objetivo é apoiar a decisão. Assim, sob esta abordagem o processo de avaliação de desempenho deve-se considerar a percepção do gestor, o alinhamento com as estratégias da organização e as variáveis do ambiente interno e externo, garantindo que as estratégias, objetivos e métricas estabelecidas estejam adequados ao ambiente da organização (Bititci, Turner \& Begemann, 2000; Bourne et al., 2000).

Behn (2003) ressalta que os gestores não devem procurar uma medida de desempenho "mágica". Em vez disso, precisam refletir sobre os objetivos organizacionais, sobre como a avaliação de desempenho poderia contribuir para o alcance desses objetivos e de que forma as medidas de desempenho poderiam ser implementadas para auxiliar nesse processo. O mesmo autor aponta que para ser eficaz a avaliação de desempenho deve possibilitar subsídio para avaliar como a organização está se saindo, orçar, controlar, motivar e promover colaboradores e equipe, comemorar resultados alcançados, aprender sobre sua atividade e aperfeiçoar o desempenho (Behn, 2003).

Nesse contexto, de acordo com Ensslin et al. (2010) a avaliação de desempenho configura-se como um processo que proporciona a construção de conhecimento no decisor sobre o contexto que se propõe a avaliar e que, de acordo com a sua percepção, identifica, organiza, mensura e integra os aspectos por ele considerados necessários e suficientes para avaliar o contexto, permitindo a visualização do impacto das decisões tomadas e o gerenciamento (Ensslin et al., 2010). 


\subsection{Avaliação de desempenho nas universidades}

As universidades estão operando em um ambiente em que a prestação de contas às partes interessadas é cada vez mais enfatizada (Agyemang \& Broadbent, 2015), entre estas, encontra-se o governo. Como o ensino superior, em muitas nações, depende fortemente de financiamento do governo, junto com as agências financiadoras, estas estão associando o financiamento das universidades com a prestação de contas sobre o desempenho delas e sobre a gestão dos recursos (Asif \& Searcy, 2014). Assim, entre outros fatores, o interesse na avaliação de desempenho das universidades tem sido resultado das pressões do setor público sobre o setor de educação (Higgins, 1989). Isso acontece porque uma das formas para demonstrar uma gestão adequada dos fundos para as partes interessadas é por meio de sistemas internos de avaliação de desempenho com base no uso de indicadores pela gestão (Asif \& Searcy, 2014).

Mesmo nas Instituições de Ensino Superior (IES) que não dependem fortemente de financiamento do governo, existe a necessidade de melhorar continuamente seu desempenho para se garantirem competitivas no mercado e garantirem um nível sustentável de financiamento. Assim, a gestão universitária, baseada no uso de indicadores de desempenho, está ganhando reconhecimento como uma ferramenta-chave para a sobrevivência das Instituições de Ensino Superior (Asif \& Searcy, 2014; Azma, 2010).

Além disso, as IES, públicas e privadas, assim como outras organizações, têm diversos tipos de recursos limitados, não só financeiros, como, por exemplo, recursos humanos, informacionais, de infraestrutura, materiais e tecnológicos. Assim, é importante gerenciar o desempenho das universidades, pelo uso de indicadores, para que os diversos recursos sejam utilizados de forma adequada (Asif \& Searcy, 2014).

Segundo Luneva (2015), a necessidade e eficiência de indicadores de desempenho em instituições de ensino estão em discussão por um longo período de tempo. Asif e Searcy (2014) ressaltam que indicadores de desempenho nas universidades são utilizados para acompanhar o desempenho de processos-chave, tais como pesquisa, ensino, extensão e gestão financeira e orçamentária.

\subsection{Avaliação de desempenho da pesquisa nas universidades}

As universidades têm, tradicionalmente, entre seus objetivos principais, a construção e a transmissão de conhecimentos (Higgins, 1989). Uma das formas de atingir esse objetivo é pela realização de pesquisas científicas. Segundo Agyemang e Broadbent (2015), a pesquisa é um processo criativo e inovador que visa à descoberta de novos conhecimentos, mas é prejudicada quando a pesquisa, dentro das universidades, torna-se impulsionada por exigências de receita. Assim, universidades precisam relembrar que o propósito da pesquisa é a geração de conhecimento, e não geração de receita (Agyemang \& Broadbent, 2015).

Segundo Prathap e Ratnaveln (2015), na maioria dos países, as universidades são as maiores contribuintes no processo de produção da pesquisa. Por isso, no ambiente em que a pesquisa e as universidades se encontram hoje, dificilmente se pode deixar o processo de investigação "não gerenciado" apenas sob responsabilidade dos acadêmicos individuais (Beerkens, 2013, p. 1679). Assim, a atuação da gestão no processo de avaliação de desempenho da pesquisa nas universidades torna-se essencial. É preciso gerenciar e controlar os processos que permitirão a criação e desenvolvimento da pesquisa para que a geração de conhecimento possa realmente ocorrer (Agyemang \& Broadbent, 2015). 
Segundo Beerkens (2013), nas últimas duas décadas, a avaliação de desempenho da pesquisa acadêmica tornou-se uma questão bastante discutida em muitos países. Korhonen, Tainio e Wallenius (2001) ressaltam que, apesar da importância visível da pesquisa em universidades, parece muito difícil avaliá-la e esse é um problema universal. Segundo Bei e Dongsheng (2014), a gestão da pesquisa universitária e a avaliação de desempenho ainda estão no estágio inicial e necessitam de desenvolvimento e aperfeiçoamento. Nesse contexto, os indicadores de desempenho são apontados como uma importante ferramenta para gestão da pesquisa universitária (Asif \& Searcy, 2014).

A literatura apresenta alguns indicadores para avaliação de desempenho da pesquisa, conforme demonstrado no Quadro 2.

Quadro 2: Indicadores encontrados na literatura para avaliar o desempenho da atividade de Pesquisa.

\begin{tabular}{|c|c|c|}
\hline Autores & Ano & \begin{tabular}{|l|} 
Indicadores Utilizados \\
\end{tabular} \\
\hline Asif e Searcy & 2014 & $\begin{array}{l}\text { Número de publicações de pesquisa (incluindo artigos de periódicos, conferências, } \\
\text { livros e monografias); número de projetos de pesquisa; porcentagem de professores } \\
\text { que ganham subsídios acadêmicos; número de projetos de tecnologia; número de } \\
\text { projetos de pesquisa que atendem às necessidades locais; porcentagem de professores } \\
\text { que frequentam conferências e seminários; impacto da investigação. }\end{array}$ \\
\hline Beerkens & 2013 & $\begin{array}{l}\text { Número total de publicações na Web of Science por pessoal acadêmico; número total } \\
\text { de livros, capítulos de livros, artigos e textos de conferências por pessoal acadêmico. }\end{array}$ \\
\hline Bei e Dongsheng & 2014 & $\begin{array}{l}\text { Número de Patentes; nível dos artigos; número de artigos; ganho da pesquisa } \\
\text { científica; eficiência no uso real do resultado; quantidade de talentos cultivados. }\end{array}$ \\
\hline $\begin{array}{l}\text { Korhonen, } \\
\text { Tainio } \\
\text { Wallenius }\end{array}$ & 2001 & $\begin{array}{l}\text { Artigos publicados em revistas internacionais; livros científicos e capítulos de livros } \\
\text { científicos publicados por editoras internacionalmente conhecidas; citações; } \\
\text { publicações superiores a um padrão mínimo de qualidade; trabalhos em anais de } \\
\text { eventos, relatórios internos, relatórios em revistas nacionais, working papers e outros } \\
\text { relatórios não publicados, trabalhos com apresentações em conferências; citações por } \\
\text { outros pesquisadores; apresentações convidadas e plenárias em conferências } \\
\text { internacionais; número de coautores estrangeiros em artigos de periódicos; teses } \\
\text { produzidas; número de estudantes de doutorado em andamento; participações em } \\
\text { conselhos editoriais; livros editados e edições especiais dos periódicos; conferências } \\
\text { científicas organizadas; participações em comissões de programa. }\end{array}$ \\
\hline Luneva & 2015 & $\begin{array}{l}\text { Número de publicações na Web of Science e Scopus (sem duplicidade) por docente; } \\
\text { número de citações por membro do corpo docente. }\end{array}$ \\
\hline $\begin{array}{l}\text { Prathap } \\
\text { Ratnavelu }\end{array}$ & 2015 & $\begin{array}{l}\text { Número total de autores da instituição em determinado período de tempo; número } \\
\text { total de documentos publicados em periódicos acadêmicos indexados na scopus; } \\
\text { número total de documentos publicados em periódicos acadêmicos indexados na } \\
\text { scopus/número total de autores da instituição; taxa de excelência (percentual da } \\
\text { produção científica que está incluída nos } 10 \% \text { dos papéis mais citados em seus } \\
\text { respectivos campos científicos). }\end{array}$ \\
\hline
\end{tabular}

Fonte: Elaborado pela autora.

\section{METODOLOGIA DA PESQUISA}

\subsection{Enquadramento metodológico}

Esta pesquisa, ao promover a geração de conhecimento sobre os aspectos relevantes que concernem à atividade de pesquisa na UM para o pró-reitor de pesquisa, classifica-se como exploratória (Richardson, 1999). Como se pretende construir um modelo personalizado para

Revista de Gestão e Secretariado-GeSec, São Paulo, v. 8, n. 2, p 76-96, Mai./Ago. 2017. 
apoiar a gestão da atividade de pesquisa da UM, utilizou-se, como procedimento técnico, o estudo de caso. Assim, a coleta de dados é de natureza primária, tendo em vista que os dados serão coletados por meio de entrevistas semiestruturadas com o pró-reitor de pesquisa da Universidade de Mindelo. Quanto à abordagem do problema, este estudo caracteriza-se como qualitativo na fase de estruturação e recomendação da metodologia Multicritério de Apoio à Decisão-Construtivista (MCDA-C) (Richardson, 1999).

\subsection{Procedimentos para seleção do material para compor o referencial teórico}

Para a seleção dos artigos que irão informar a construção do referencial teórico, utilizou-se o Knowledge Development Process-Constructivist (Proknow-C). Trata-se de um processo estruturado e sistematizado, constituído de quatro etapas: 1) seleção de portfólio bibliográfico (PB); 2) análise bibliométrica; 3) análise sistêmica; e 4) formulação de perguntas e objetivos de pesquisa. Esse instrumento também foi operacionalizado nas pesquisas de Thiel, Ensslin e Ensslin (2017), Valmorbida e Ensslin (2015), Dutra, RipollFeliu, Fillol, Ensslin \& Ensslin, L. (2015), Cardoso et al. (2015), Ensslin et al. (2014), Valmorbida et al. (2014), Ensslin, Ensslin e Pinto (2013), Waiczyk \& Ensslin, 2013, entre outras.

Nesta pesquisa, apenas a primeira etapa do Proknow-C foi operacionalizada, com o objetivo de selecionar artigos alinhados ao fragmento da literatura referente à avaliação de desempenho da pesquisa de universidades, segundo a percepção dos pesquisadores. Essa etapa é feita por meio da "seleção do banco de artigos bruto", da "filtragem do banco de artigos" e é finalizada com o "teste de representatividade dos artigos do Portfólio Bibliográfico". As demais etapas não foram operacionalizadas por não estarem alinhadas ao objetivo da pesquisa de estruturação de um modelo de avaliação de desempenho (a etapa 1 dará subsídios para identificação de indicadores e para a compreensão de como a avaliação de desempenho da atividade da pesquisa vem sendo trabalhada na literatura).

A seleção do banco de artigos bruto envolve a definição dos eixos de pesquisa, das palavras-chave, das bases de dados e a busca dos artigos. Nesta pesquisa, foram utilizados os seguintes eixos e palavras-chave: Avaliação de Desempenho (Performance Measurement; Performance Mesure; Performance Management; Performance Evaluation; Performance Assessment), Universidade (Higher Education; Universit*; Undergraduate; e College) e Pesquisa (Research*). As palavras utilizadas com asteriscos são para buscar os diversos sufixos do radical. As bases de dados utilizadas para a busca dos artigos foram: Proquest, Web of Science, EBSCO, Science Direct, Scopus e Engineering Village. A busca foi realizada no dia 26 de janeiro de 2016 e resultou em um total de 3.977 publicações distribuídas entre as bases de dados (sem delimitação temporal).

A etapa de filtragem do banco de dados inicia com a eliminação dos artigos repetidos. Com o auxílio do EndNote ${ }^{\circledR}$ X7, dos 3.977 artigos, foram identificados 667 artigos em duplicidade ou que não eram artigos científicos, os quais foram excluídos do processo. Dessa maneira, o processo seguiu com 3.310 artigos. Destes, verificou-se o alinhamento do título dos artigos, dos quais 235 artigos foram considerados com o título alinhado. Após a verificação do alinhamento do título, fez-se o reconhecimento científico dos artigos com base nas suas citações, por meio do Google Scholar, para posterior verificação do alinhamento do resumo. Porém os autores desta pesquisa optaram por verificar o resumo de todos os artigos, sem delimitá-los pelo reconhecimento científico. Assim, verificou-se o alinhamento do resumo dos 235 artigos com título alinhado. Destes artigos, 57 estavam com o resumo alinhado e apenas 34 artigos estavam disponíveis gratuitamente. Dos artigos disponíveis, nove 
estavam integralmente alinhados ao objetivo desta pesquisa, segundo a percepção dos pesquisadores. Assim, concluiu-se a etapa da filtragem do banco de artigos com nove artigos e passou-se para etapa do teste de representatividade dos artigos do Portfólio Bibliográfico.

O teste de representatividade foi realizado por meio da análise das referências bibliográficas dos nove artigos integralmente alinhados. Nessa análise, verificou-se o alinhamento de 269 referências, onde 10 referências foram consideradas com o título alinhado. Das 10 referências, três estavam com o resumo alinhado, e apenas duas estavam disponíveis gratuitamente. Destas, nenhuma estava integralmente alinhada com o objetivo da pesquisa, portanto não foram incluídas no Portfólio. Dessa forma, concluiu-se a Seleção do Portfólio Bibliográfico com nove artigos que representaram o fragmento da literatura referente à Avaliação de Desempenho da pesquisa das universidades. O Quadro 1 apresenta os artigos que compõem o Portfólio Bibliográfico (PB) desta pesquisa.

Quadro 1: Artigos que compõem o portfólio bibliográfico.

\begin{tabular}{|c|c|c|c|}
\hline Autores & Título & Periódico & Ano \\
\hline $\begin{array}{l}\text { Agyemang, G. } \\
\text { Broadbent, J. }\end{array}$ & $\begin{array}{l}\text { Management control systems and research } \\
\text { management in universities: An empirical and } \\
\text { conceptual exploration }\end{array}$ & $\begin{array}{l}\text { Accounting Auditing } \\
\text { and Accountability }\end{array}$ & 2015 \\
\hline $\begin{array}{l}\text { Asif, M. } \\
\text { Searcy, C. }\end{array}$ & $\begin{array}{l}\text { A composite index for measuring performance in } \\
\text { higher education institutions }\end{array}$ & $\begin{array}{l}\text { International Journal } \\
\text { of Quality \& Reliability } \\
\text { Management }\end{array}$ & 2014 \\
\hline Azma, F. & $\begin{array}{l}\text { Qualitative Indicators for the evaluation of } \\
\text { universities performance }\end{array}$ & $\begin{array}{l}\text { Procedia - Social and } \\
\text { Behavioral Sciences }\end{array}$ & 2010 \\
\hline Beerkens, M. & $\begin{array}{l}\text { Facts and fads in academic research management: } \\
\text { The effect of management practices on research } \\
\text { productivity in Australia }\end{array}$ & Research Policy & 2013 \\
\hline $\begin{array}{l}\text { W. Bei } \\
\text { L. Dongsheng }\end{array}$ & $\begin{array}{l}\text { The Performance Evaluation of University Scientific } \\
\text { Research Project Management Based on the FAHP }\end{array}$ & $\begin{array}{l}\text { Journal of Digital } \\
\text { Information } \\
\text { Management }\end{array}$ & 2014 \\
\hline Higgins, J. C. & Performance measurement in universities & $\begin{array}{l}\text { European Journal of } \\
\text { Operational Research }\end{array}$ & 1989 \\
\hline $\begin{array}{l}\text { Korhonen, P. } \\
\text { Tainio, R. } \\
\text { Wallenius, J. }\end{array}$ & Value efficiency analysis of academic research & $\begin{array}{l}\text { European Journal of } \\
\text { Operational Research }\end{array}$ & 2001 \\
\hline Luneva, E. V. & $\begin{array}{l}\text { Key performance indicators (KPI) system in } \\
\text { education }\end{array}$ & Asian Social Science & 2015 \\
\hline $\begin{array}{l}\text { Prathap, G. } \\
\text { Ratnavelu, K. }\end{array}$ & $\begin{array}{l}\text { Research performance evaluation of leading higher } \\
\text { education institutions in Malaysia }\end{array}$ & Current Science & 2015 \\
\hline
\end{tabular}

Fonte: Elaborado pelos autores (2016).

Dessa forma, os artigos selecionados e apresentados na Tabela 1 serão utilizados na construção do referencial teórico referente aos fragmentos "Avaliação de Desempenho nas Universidades" e "Avaliação de Desempenho da Pesquisa nas Universidades".

\subsection{Instrumento para construção do modelo multicritério construtivista e procedimentos para coleta dos dados}

Para construir um modelo de avaliação de desempenho multicritério construtivista para apoiar a gestão e as tomadas de decisões relacionadas à atividade de pesquisa da Universidade de Mindelo (UM), será utilizada, como instrumento de intervenção, a Metodologia

Revista de Gestão e Secretariado-GeSec, São Paulo, v. 8, n. 2, p 76-96, Mai./Ago. 2017. 
Multicritério de Apoio à Decisão-Construtivista (MCDA-C), desenvolvida pelo Laboratório de Metodologias Multicritério de Apoio à Decisão (LabMCDA), da Universidade Federal de Santa Catarina (UFSC). Essa metodologia também foi operacionalizada nos estudos de Ensslin et al. (2017); Valmorbida et al. (2015); Longaray e Ensslin (2015); Ensslin et al. (2015); Ensslin et al. (2013); Lacerda, Ensslin e Ensslin (2011); Ensslin et al. (2010); Ensslin, Dutra e Ensslin (2000), entre outros.

A metodologia MCDA-C, de acordo com Ensslin et al. (2010, p.130), tem como sua "principal vocação o processo de desenvolver o conhecimento do decisor sobre o contexto". Essa geração de conhecimento é permitida por meio da operacionalização das três fases da metodologia MCDA-C, conforme Figura 1.

Figura 1: Fases da Metodologia Multicritério de Apoio à Decisão Construtivista (MCDA-C).

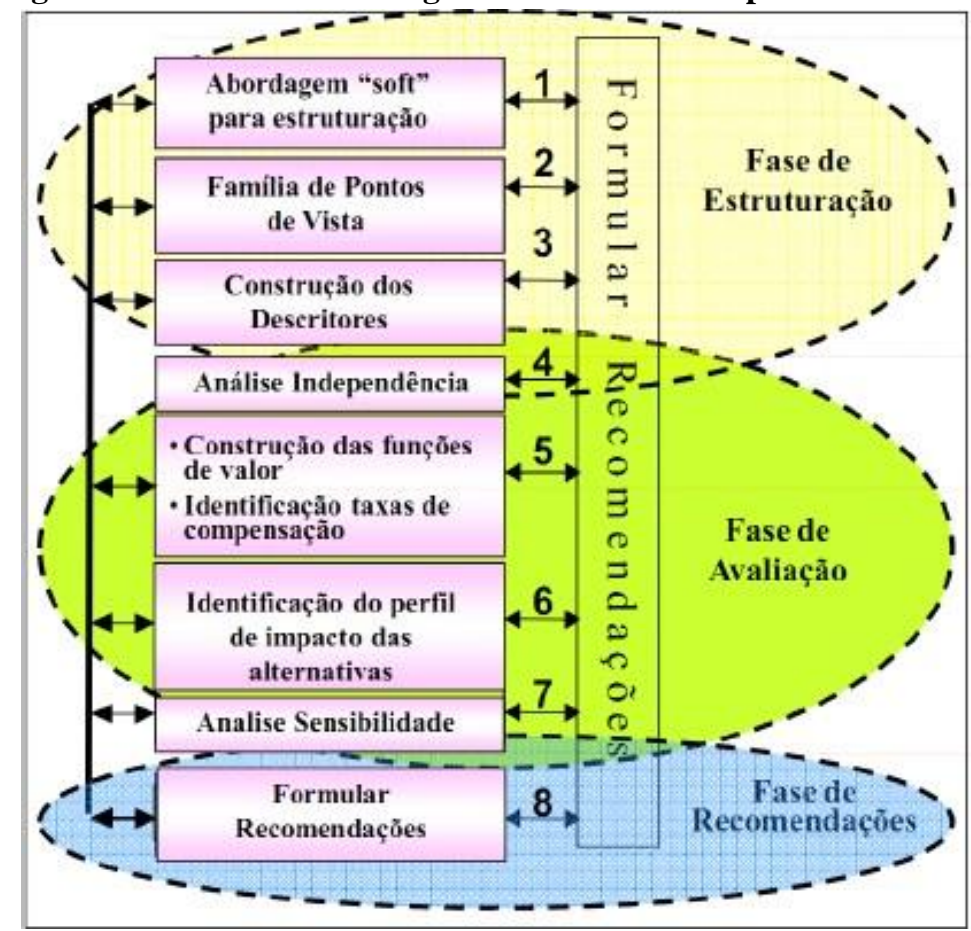

Fonte: Adaptado de Ensslin, Dutra e Ensslin (2000, p. 81).

A fase de estruturação, de acordo com Valmorbida et al. (2015), tem como objetivo a geração de conhecimento do contexto para o decisor, identificando quais aspectos são importantes segundo a sua percepção, de modo que suas preferências sejam incorporadas na construção do modelo. Assim, essa metodologia, ao ter uma abordagem construtivista, busca gerar o conhecimento no decisor a respeito do contexto em que ele está inserido e quanto ao problema por ele percebido. Esse conhecimento é obtido por meio de diversas entrevistas semiestruturadas que buscam identificar aspectos relevantes do contexto segundo a percepção do decisor, com base na fala do gestor (Ensslin et al., 2010). Nessa fase, após a identificação dos aspectos importantes para serem geridos, indicadores são construídos e, também, a forma de medi-los, e os níveis de referência de cada situação são identificados (Valmorbida et al., 2015).

Após a conclusão da fase de estruturação, onde são identificados os aspectos relevantes, necessários e suficientes segundo a percepção do decisor, tem-se contruído um modelo para avaliar o contexto, que considera tais aspectos (Ensslin et al., 2010). Na fase de avaliação, realiza-se a coleta de dados e mensuração dos indicadores construídos na fase de estruturação; 
ou seja, as escalas ordinais (qualitativas) são transformadas em escalas cardinais (quantitativas) (Valmorbida et al., 2015). São identificadas, também, as taxas de compensão que permitem a agregação dos aspectos para obtenção da avaliação global. Além disso, faz-se o julgamento das diferenças de atratividade entre os níveis de desempenho e a compensação dos indicadores para, então, identificar o status quo das alternativas (Ensslin et al., 2013). Essa etapa não será operacionalizada neste trabalho, uma vez que o pró-reitor optou por neste primeiro momento - focar na geração de conhecimento de quais aspectos são importantes e respondem pela pesquisa da Universidade de Mindelo.

Já a fase de recomendações busca auxiliar o decisor na identificação de ações de melhoria do estado atual do perfil de desempenho, observado no contexto gerido, em cada indicador. Pode-se então identificar as consequências das ações em cada nível de desempenho e na avaliação global do modelo (Ensslin et al. 2013). Essa etapa também é realizada pela interação entre o gestor e os facilitadores, visando identificar oportunidades para melhoria dos aspectos considerados importantes e podem, em curto ou longo prazo, comprometer o desempenho. Tendo identificado esses aspectos, pode-se desenvolver estratégias para aperfeiçoamento do desempenho observado.

Assim, nessa pesquisa apenas as fases de estruturação e recomendações serão operacionalizadas neste estudo e o passo a passo será demonstrado na seção de resultados.

\section{RESULTADOS}

Nesta seção, apresenta-se a construção do modelo de avaliação de desempenho multicritério construtivista da atividade de pesquisa da Universidade de Mindelo, sediada em Cabo Verde, na África.

\subsection{Construção do modelo de avaliação de desempenho utilizando a metodologia MCDA-C}

O modelo de avaliação de desempenho da atividade de pesquisa da Universidade de Mindelo foi construído de acordo com a percepção do pró-reitor de pesquisa (decisor) com base nas informações obtidas em diversas entrevistas estruturadas realizadas. A escolha do pró-reitor como decisor deve-se ao fato de ele ser o responsável e a pessoa com as competências necessárias para tomar decisões a respeito da pesquisa da UM.

Na metodologia MCDA-C, a fase de estruturação busca a geração de conhecimento no decisor a respeito do contexto e do problema por ele vivenciado para que ele evidencie quais são suas preferências e que aspectos são importantes para serem medidos e incorporados ao modelo (Ensslin et al. 2015). Assim, com base na geração do conhecimento, definiu-se o seguinte rótulo para o modelo "Avaliação de Desempenho da Atividade de Pesquisa da Universidade de Mindelo" e identificaram-se os atores envolvidos no contexto, conforme Quadro 3. 
Quadro 3: Atores do Contexto.

\begin{tabular}{|l|l|l|}
\hline \multirow{4}{*}{ Stakeholders } & Decisor & Pró-reitor de Pesquisa da UM \\
\cline { 2 - 3 } & \multirow{4}{*}{ Intervenientes } & Reitor da UM \\
\cline { 3 - 3 } & & Professores \\
\cline { 2 - 3 } & Funcionários \\
\cline { 2 - 3 } Agidos & Facilitadores & Autores do trabalho \\
\hline \multirow{2}{*}{ Agides } & Alunos \\
\cline { 2 - 2 } & Professores \\
\cline { 2 - 2 } & População de Cabo Verde \\
\hline
\end{tabular}

Fonte: Dados da Pesquisa (2016).

Após a definição do rótulo, identificaram-se os Elementos Primários de Avaliação (EPAs) que são características ou propriedades, que o decisor julga importante e/ou que impactam seus valores e preferências a respeito do contexto (Ensslin et al., 2013; Ensslin et al., 2010). Com base na identificação dos EPAs, constroem-se os conceitos que indicam a direção de preferência, relacionada ao EPA e acompanhada por seu oposto psicológico (mínimo aceitável do objetivo subjacente), representado pelas reticências (...) que devem ser lidas como ao invés de. Um EPA pode ter mais de um conceito (Ensslin et al., 2013; Ensslin et al., 2010; Ensslin; Neto \& Noronha, 2001). Nessa etapa, ao todo, 39 EPAs e 64 conceitos foram construídos, sendo que alguns deles estão apresentados no Quadro 4.

Quadro 4: EPAs e conceitos construídos.

\begin{tabular}{|c|c|}
\hline EPAs & Conceitos \\
\hline Visibilidade & $\begin{array}{l}251 \text { - Dar visibilidade à instituição (UM) ... Perder a oportunidade de dar visibilidade à } \\
\text { instituição. }\end{array}$ \\
\hline Sustentabilidade & $\begin{array}{l}252 \text { - Contribuir para o desenvolvimento sustentável da cidade/país ... Comprometer a } \\
\text { imagem da instituição. }\end{array}$ \\
\hline Resultados & $\begin{array}{l}253 \text { - Divulgar os resultados das pesquisas ... Perder a oportunidade de dar visibilidade à } \\
\text { instituição. }\end{array}$ \\
\hline Patentes & 254 - Registrar as patentes das pesquisas ... Deixar de ter registro de patentes. \\
\hline Parcerias & 255 - Gerar novos negócios/empresas ... Deixar de ampliar a rede de parceiros. \\
\hline Artigos & $\begin{array}{l}256 \text { - Fomentar a publicação de artigos em periódicos (nacionais e internacionais) ... } \\
\text { Perder a oportunidade de promover a pesquisa institucional. }\end{array}$ \\
\hline Artigos & $\begin{array}{l}257 \text { - Fomentar a divulgação de trabalhos em eventos ... Perder a oportunidade de } \\
\text { promover a pesquisa institucional. }\end{array}$ \\
\hline $\begin{array}{l}\text { Trabalhos de } \\
\text { conclusão de curso }\end{array}$ & $\begin{array}{l}258 \text { - Finalizar e divulgar os trabalhos de conclusão de curso }(\mathrm{L}, \mathrm{M}, \mathrm{D}) \text {... Não finalizar os } \\
\text { trabalhos de conclusão de curso. }\end{array}$ \\
\hline Visibilidade & $\begin{array}{l}259 \text { - Potencializar a expansão da UM ... Perder a oportunidade de dar visibilidade à } \\
\text { instituição. }\end{array}$ \\
\hline Seminários/Eventos & $\begin{array}{l}260 \text { - Fomentar a apresentação de trabalhos em eventos ... Perder a oportunidade de } \\
\text { promover a pesquisa institucional. }\end{array}$ \\
\hline Seminários/Eventos & $\begin{array}{l}261 \text { - Fomentar a publicação de trabalhos em eventos ... Perder a oportunidade de } \\
\text { promover a pesquisa institucional. }\end{array}$ \\
\hline Corpo docente & $\begin{array}{l}262 \text { - Potencializar o reconhecimento do corpo docente ... Perder a oportunidade de dar } \\
\text { visibilidade à instituição. }\end{array}$ \\
\hline Parcerias & 263 - Potencializar parcerias ... Sofrer pela falta de recursos. \\
\hline Corpo docente & 264 - Potencializar captação de recursos ... Sofrer pela falta de recursos. \\
\hline
\end{tabular}

Fonte: Dados da Pesquisa (2016).

De acordo com Ensslin et al. (2010, p. 134), após construídos os conceitos, "que representam preocupações estratégicas equivalentes, segundo a percepção do decisor, podem ser agrupados em Áreas de Preocupação". Nesta pesquisa, constatou-se que os conceitos

Revista de Gestão e Secretariado-GeSec, São Paulo, v. 8, n. 2, p 76-96, Mai./Ago. 2017. 
estavam associados a cinco áreas: capacitação; estruturação da pesquisa; captação de recursos; operacionalização da pesquisa; e resultados da pesquisa, formando a estrutura hierárquica de valor com os pontos de vistas fundamentais (PVFs). Essa estrutura foi testada quanto à sua necessidade e suficiência.

Com os conceitos agrupados em áreas de preocupação, é possível criar mapas cognitivos que permitem a expansão do conhecimento para o decisor, onde conceitos podem ser incluídos, bem como descartados, o que faz parte do processo de geração de conhecimento permitido pela metodologia MCDA-C. A Figura 2 representa o mapa cognitivo para PVF "Resultados da Pesquisa".

Figura 2: Mapa Cognitivo para o PVF "Resultados da Pesquisa”.

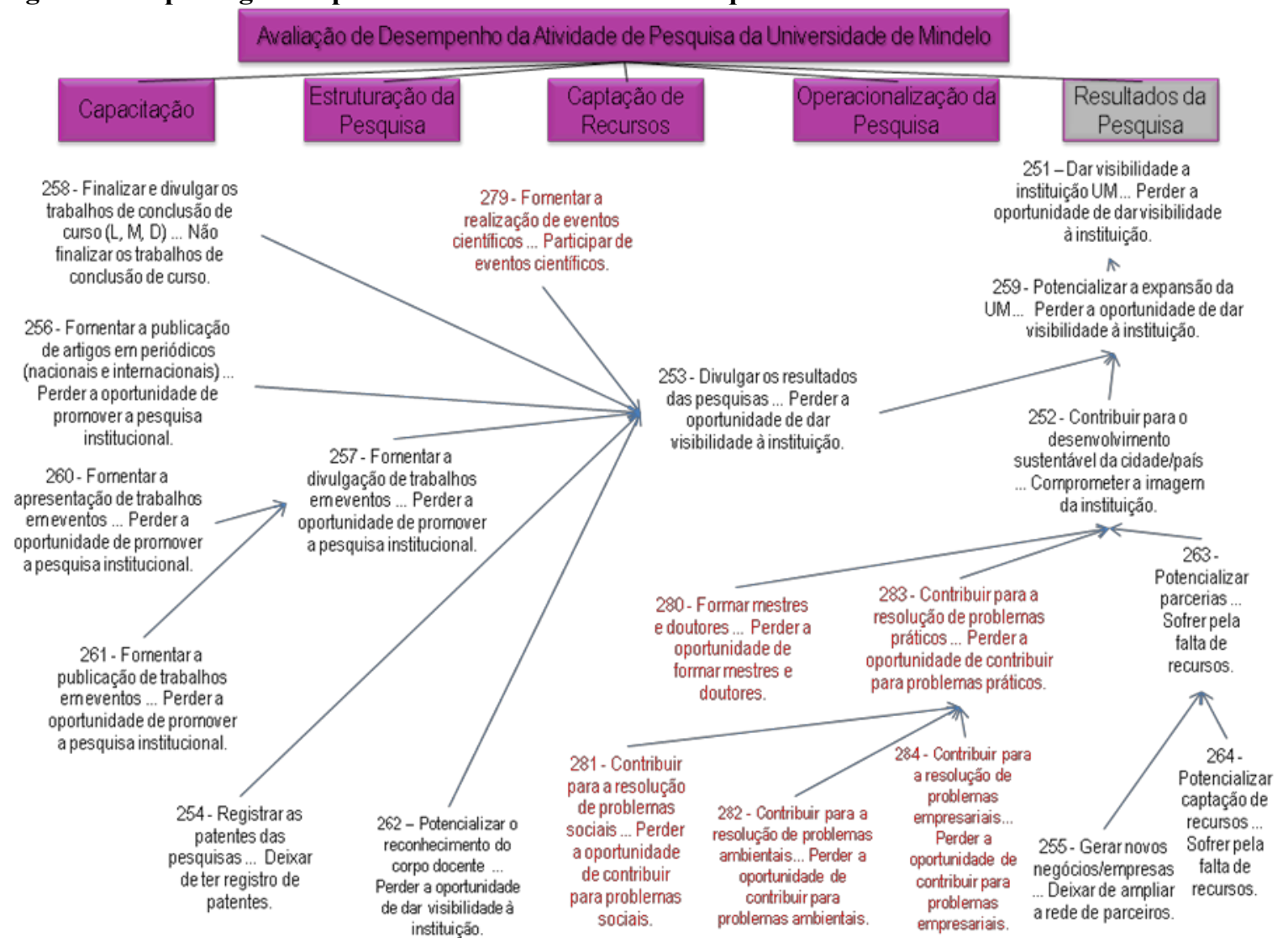

Fonte: Dados da Pesquisa (2016).

Para facilitar a análise dos mapas cognitivos, estes foram divididos em clusters e subclusters. Para cada cluster, um nome que representa o foco de interesse do decisor foi associado (Ensslin et al., 2013; Ensslin et al., 2010; Ensslin; Neto \& Noronha, 2001). Após o agrupamento em clusters e subclusters, origina-se a Árvore de Valor com os Pontos de Vistas Elementares (PVEs), os quais são apresentados na Figura 3. 
Figura 3: Árvore de Valor com os Pontos de Vistas Elementares (PVEs).

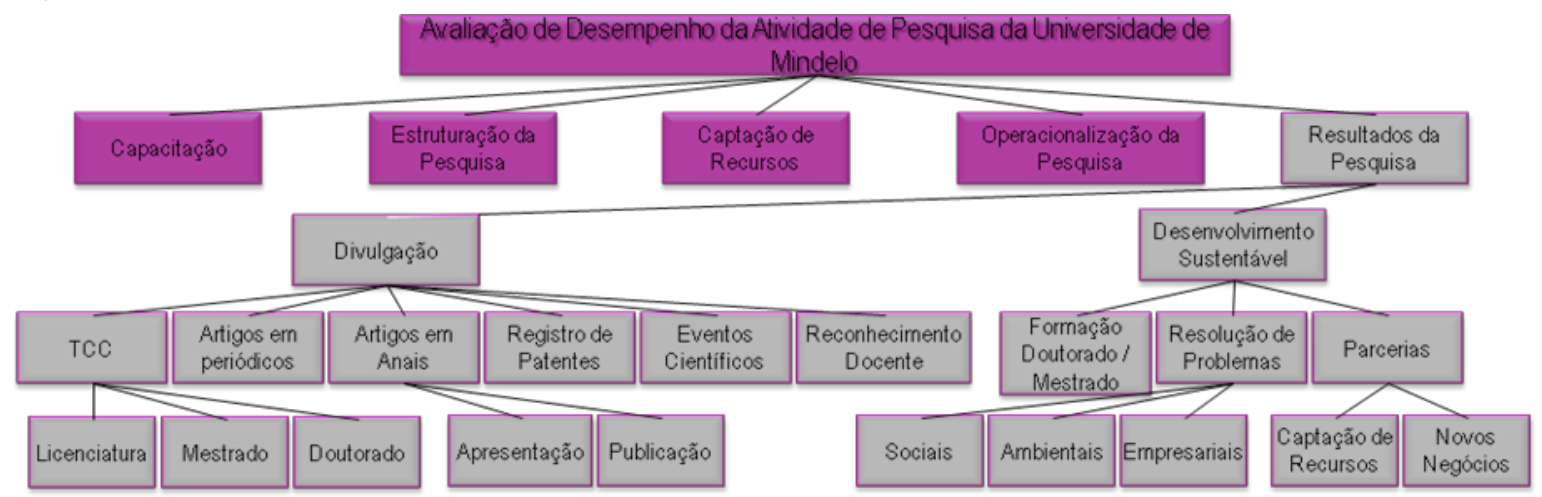

Fonte: Dados da pesquisa (2016).

Para cada um dos PVEs foram construídas as escalas ordinais (na metodologia MCDA$\mathrm{C}$ denominadas por descritores) que possibilitam a medição do desempenho das propriedades de cada PVE que operacionalizam os objetivos apresentados nos mapas cognitivos (Ensslin et al., 2015). O próximo passo consiste no estabelecimento dos níveis de referência "Bom" e "Neutro" que permitem a comparação de desempenho entre os descritores (escalas) (Ensslin; Dutra \& Ensslin, 2000). Todos os desempenhos que impactarem em um dos pontos da escala (descritor) acima do nível de referência "Bom" são considerados de excelência; os que impactarem abaixo do nível de referência "Neutro" são considerados de desempenho comprometedor; e, aqueles que impactam entre os dois níveis de referência são considerados como de desempenho de competitivo (de mercado/esperado) (Ensslin; Neto \& Noronha, 2001). A partir dos níveis de referência definidos pode-se traçar o perfil de desempenho da UM, conforme Figura 4. 
Figura 4: Estrutura Hierárquica de Valor e Descritores construídos - PVF "Resultados da Pesquisa".
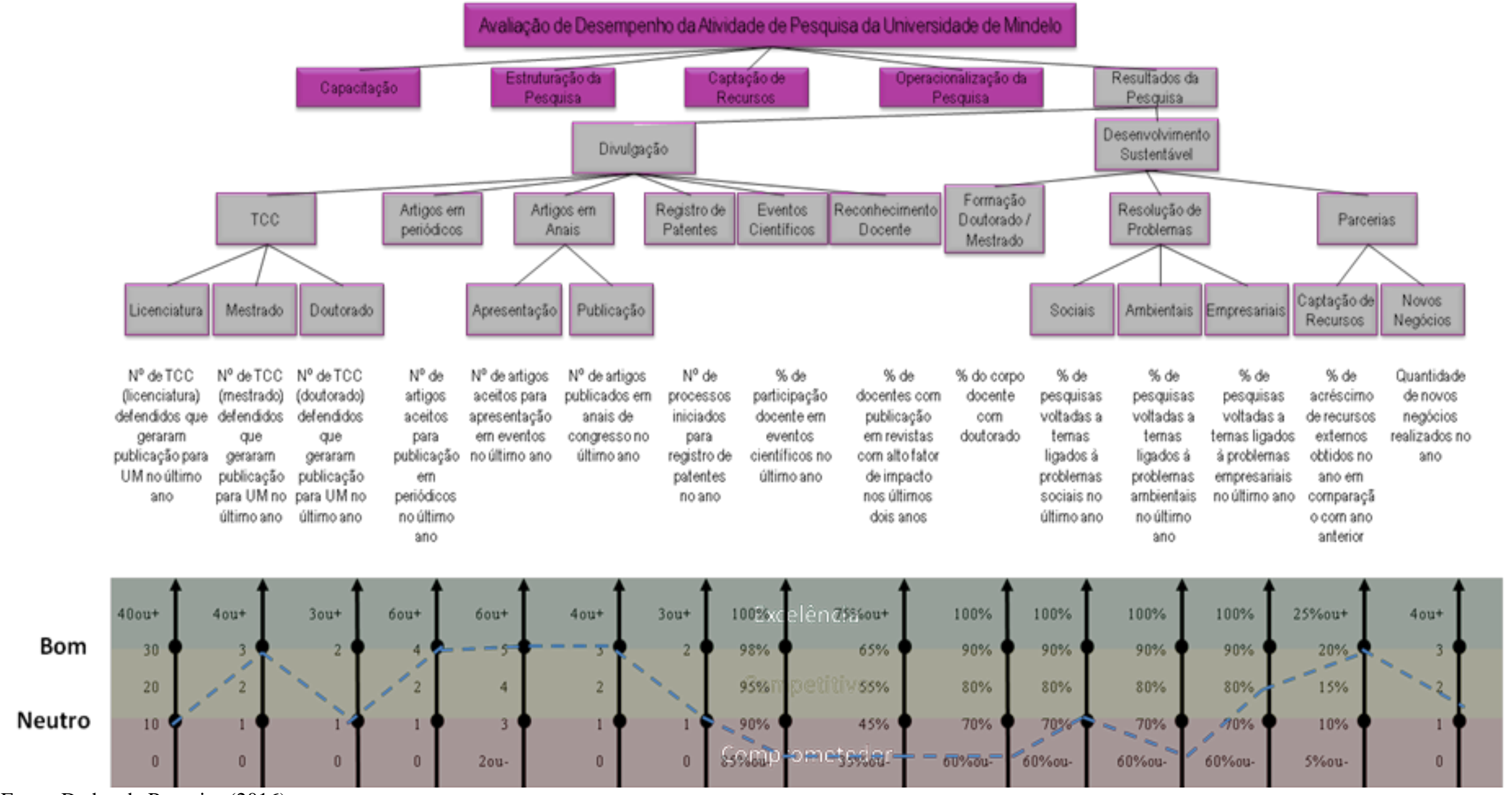

Fonte: Dados da Pesquisa (2016).

Revista de Gestão e Secretariado-GeSec, São Paulo, v. 8, n. 2, p 76-96, Mai./Ago. 2017. 
De acordo com a Figura 4, pode-se perceber que, para o PVF "Resultados da Pesquisa", 15 indicadores foram construídos que permitem avaliar a preocupação subjacente à avaliação da área de pesquisa, no que tange aos resultados proporcionados pela UM nessa questão. Assim, encerrou-se a Fase de Estruturação do modelo de avaliação de desempenho pretendido.

Após a construção dos indicadores, notou-se divergência entre os indicadores construídos nessa pesquisa, com base na percepção do decisor, e os indicadores encontrados na literatura consultada. Percebe-se que, conforme já constatado, os seguintes indicadores número de artigos publicados em periódicos, trabalhos em anais de eventos e patentes -, identificados na literatura, também foram observados como importantes na Universidade de Mindelo. No entanto, os indicadores da literatura referentes ao número de citações de artigos dos docentes; número de conclusões de doutorado; palestras científicas; direitos autorais; livros científicos; número de orientandos de doutorado; e ao percentual da receita gerada com base nas pesquisas científicas e pesquisa e desenvolvimento no montante total de receitas das universidades não se referem a aspectos específicos da gestão da pesquisa da UM.

Isso acontece em virtude do viés construtivista da metodologia MCDA-C que considera que o modelo desenvolvido é personalizado ao contexto de aplicação. Assim, as peculiaridades e características da Universidade do Mindelo e a percepção do pró-reitor foram consideradas na construção dos indicadores na fase de estruturação do modelo.

Após a construção dos indicadores, com a identificação do perfil de desempenho obtido pela universidade, objeto deste estudo de caso, denominada de status quo (linha tracejada azul na Figura 4), é possível identificar os aspectos que a gestão da pesquisa está com desempenho Bom, e aqueles em que há necessidade de aperfeiçoamento para melhoria do desempenho individual e global. Essa atividade é realizada na Fase de Recomendações.

$\mathrm{Na}$ Fase de Recomendações, os indicadores que apresentavam um desempenho no nível comprometedor foram identificados, e ações de melhorias foram propostas para o aperfeiçoamento do desempenho da atividade de pesquisa da Universidade de Mindelo. Os indicadores de desempenho que se encontravam no nível comprometedor foram: percentual de participação docente em eventos científicos no último ano; percentual de docentes com publicação em revistas de alto fator de impacto nos últimos dois anos; percentual do corpo docente com doutorado; e percentual de pesquisas voltadas a temas ligados a problemas ambientais no último ano. A Figura 5 apresenta as ações de melhorias propostas para alavancar o desempenho da atividade de pesquisa nesses quatro indicadores.

Figura 5: Ações de aperfeiçoamento do desempenho de pesquisa da UM.

\begin{tabular}{|l|l|}
\hline PVE & Eventos Científicos \\
\hline Indicador & Percentual de participação docente em eventos científicos no último ano \\
\hline Ações Propostas \\
\hline Buscar informações e divulgar a realização de eventos científicos \\
\hline Incentivar a submissão de trabalhos aos eventos \\
\hline Fornecer auxílio financeiro para a participação em eventos científicos \\
\hline Abater as horas de atividades administrativas pelas horas de participação em eventos \\
\hline Responsável & Pró-reitor de pesquisa \\
\hline Prazo de Atendimento & Diariamente \\
\hline Impacto no descritor & Passaria do nível comprometedor para o nível competitivo \\
\hline PVE & Reconhecimento Docente \\
\hline Indicador & $\begin{array}{l}\text { Percentual de docentes com publicação em revistas com alto fator de impacto nos } \\
\text { últimos dois anos }\end{array}$ \\
\hline
\end{tabular}




\begin{tabular}{|c|c|c|}
\hline \multicolumn{3}{|c|}{ Ações Propostas } \\
\hline \multicolumn{3}{|c|}{ Abater as horas-aula em sala pelas horas de dedicação a realização de pesquisas } \\
\hline \multicolumn{3}{|c|}{ Fornecer e fomentar laboratórios de pesquisa } \\
\hline \multicolumn{3}{|c|}{ Incentivar a criação de grupos de pesquisa } \\
\hline \multicolumn{2}{|c|}{ Responsável } & Pró-reitor de pesquisa \\
\hline \multirow{2}{*}{\multicolumn{2}{|c|}{$\begin{array}{l}\text { Prazo de Atendimento } \\
\text { Impacto no descritor }\end{array}$}} & Diariamente \\
\hline & & Passaria do nível comprometedor para o nível competitivo \\
\hline PVE & \multicolumn{2}{|c|}{ Formação Doutorado / Mestrado } \\
\hline Indicador & \multicolumn{2}{|c|}{ Percentual do corpo docente com doutorado } \\
\hline \multicolumn{3}{|c|}{ Ações Propostas } \\
\hline \multicolumn{3}{|c|}{ Possibilitar o afastamento de docentes para doutorado. } \\
\hline \multicolumn{3}{|c|}{ Fornecer acréscimo salarial para docentes doutores. } \\
\hline \multicolumn{2}{|c|}{ Responsável } & Pró-reitor de pesquisa/Pró-reitor financeiro. \\
\hline \multicolumn{2}{|c|}{ Prazo de Atendimento } & Anualmente. \\
\hline \multicolumn{2}{|c|}{ Impacto no descritor } & Passaria do nível comprometedor para o nível competitivo. \\
\hline PVE & \multicolumn{2}{|c|}{ Pesquisas para solução de problemas Ambientais } \\
\hline Indicador & \multicolumn{2}{|c|}{$\begin{array}{l}\text { Percentual de pesquisas voltadas a temas ligados a problemas ambientais no último } \\
\text { ano. }\end{array}$} \\
\hline \multicolumn{3}{|c|}{ Ações Propostas } \\
\hline \multicolumn{3}{|c|}{ Incentivar os acadêmicos à realização de pesquisas voltadas aos problemas ambientais. } \\
\hline \multicolumn{3}{|c|}{ Fornecer disciplinas que abordem os problemas ambientais e as possibilidades de pesquisas na área. } \\
\hline \multicolumn{3}{|c|}{ Incentivar a participação em eventos que abordem a temática. } \\
\hline \multicolumn{2}{|c|}{ Responsável } & Pró-reitor de pesquisa/docentes vinculados à Pós-graduação. \\
\hline \multirow{2}{*}{\multicolumn{2}{|c|}{$\begin{array}{l}\text { Prazo de Atendimento } \\
\text { Impacto no descritor }\end{array}$}} & Semestralmente. \\
\hline & & Passaria do nível comprometedor para o nível competitivo. \\
\hline
\end{tabular}

Fonte: Dados da Pesquisa (2016).

Observa-se que os descritores com desempenho comprometedor se referem à qualificação e atualização dos docentes e à realização de pesquisas científicas. Dessa forma, essas situações, agora, apresentam oportunidades de melhoria, o que contribuirá para o aperfeiçoamento da atividade de pesquisa da UM e para a geração de conhecimento para a comunidade científica.

Algumas ações propostas dependem somente da pró-reitoria da pesquisa; para outras, há necessidade de colaboração da equipe financeira e da reitoria, pois envolvem ações que impactam outros departamentos. No entanto, como interferem também no desempenho da pró-reitoria de pesquisa, cabe ao pró-reitor buscar uma solução junto com os demais departamentos.

\section{CONCLUSÃO}

A avaliação de desempenho das universidades está se tornando cada vez mais indispensável para a sobrevivência das Instituições de Ensino Superior, uma vez que é necessário que a gestão universitária acompanhe o desempenho de seus processos-chave, tais como pesquisa, ensino, extensão e gestão financeira e orçamentária, para que seus objetivos sejam atingidos.

Revista de Gestão e Secretariado-GeSec, São Paulo, v. 8, n. 2, p 76-96, Mai./Ago. 2017. 
Entre os processos-chave, destaca-se a necessidade da avaliação da atividade de pesquisa, visto que um dos objetivos das universidades é a geração de conhecimento, proporcionado por meio da pesquisa científica. Nesse contexto, esta pesquisa teve por objetivo a estruturação de um modelo de avaliação de desempenho para apoiar a gestão da atividade de pesquisa da Universidade de Mindelo (UM), sediada em Cabo Verde, na África. Para atender ao objetivo, utilizou-se, como instrumento de intervenção, a Metodologia Multicritério de Apoio à Decisão-Construtivista (MCDA-C). Assim, para a construção do modelo de avaliação de desempenho ad hoc para a UM, entrevistas semiestruturadas foram realizadas, onde identificaram-se 39 Elementos Primários de Avaliação (EPAs), os quais deram origem a 64 conceitos que foram agrupados em cinco áreas de preocupação: capacitação; estruturação da pesquisa; captação de recursos; operacionalização da pesquisa; e resultados da pesquisa, formando a estrutura hierárquica de valor com os pontos de vistas fundamentais (PVFs). Após o agrupamento, mapas cognitivos foram criados que permitiram que novos conceitos fossem incluídos e alguns descartados por terem contribuído para expansão do conhecimento do pró-reitor.

Depois da criação dos mapas, os conceitos foram divididos em clusters e subclusters e deram origem a Árvore de Valor com os Pontos de Vistas Elementares (PVEs). Para os PVEs foram construídos descritores (escalas ordinais qualitativas) que permitiram a transmissão da informação do que é importante, para a UM, em cada uma dessas escalas, bem como sua ordem de preferência. Na sequência, os níveis de referência (com o nível Bom e o nível Neutro) foram definidos e o perfil de desempenho (status quo) da UM foi identificado.

Com a definição do status quo, foi possível identificar os aspectos que a gestão da pesquisa apresentava como um desempenho Bom e aqueles que apresentavam um desempenho comprometedor. Os aspectos da gestão que estavam com desempenho comprometedor são: percentual de participação docente em eventos científicos no último ano; percentual de docentes com publicação em revistas de alto fator de impacto nos últimos dois anos; percentual do corpo docente com doutorado; e percentual de pesquisas voltadas a temas ligados a problemas ambientais no último ano. Para esses aspectos, tendo em vista a necessidade de aperfeiçoamento para melhoria dos desempenhos individual e global, as ações de melhoria foram propostas para alavancar o desempenho da atividade de pesquisa nesses quatro indicadores.

Além disso, neste estudo, identificou-se, ao realizar um cotejamento dos indicadores construídos neste estudo com os encontrados na literatura, que a maior parte dos indicadores propostos pela literatura não foram incorporados ao modelo estruturado nesta pesquisa. As divergências justificam-se pelo viés construtivista da metodologia que tem como premissa a construção de conhecimento no decisor, considerando a sua percepção para a identificação dos aspectos, por ele julgados como necessários e suficientes, para avaliar o contexto de aplicação (no caso deste estudo, a Universidade do Mindelo). Assim, este estudo estruturou um modelo personalizado para a UM, considerando suas características e peculiaridades.

$\mathrm{Na}$ percepção dos autores deste estudo, a construção do modelo personalizado, para avaliar a atividade de pesquisa da Universidade de Mindelo, além de dar subsídios para antever a avaliação institucional externa, apoiará a tomada de decisão da pró-reitoria de pesquisa, contribuindo para o desenvolvimento da pesquisa científica, para geração de conhecimento dos pesquisadores em geral, centralmente, dos pesquisadores de Mindelo. Isso corrobora com o exposto por Agyemang e Broadbent (2015) que ressaltam que é preciso gerenciar os processos que permitirão a realização pesquisa para que a geração de conhecimento possa realmente ocorrer.

Assim, nesta pesquisa é demonstrada uma forma de avaliar a pesquisa dentro das universidades, fato descrito por Korhonen, Tainio e Wallenius (2001) como de difícil 
operacionalização, sendo um problema universal. Entretanto, ressalta-se que este estudo se limita à construção de um modelo personalizado (ad hoc) para apoiar a gestão da Universidade de Mindelo, segundo a percepção do pró-reitor de pesquisa, nas decisões a serem tomadas. Dessa forma, para a replicação do estudo é preciso adaptações que devem considerar o contexto único do objeto a ser replicado; ou seja, os aspectos importantes para a UM não necessariamente são importantes e contribuirão para a melhoria do desempenho de outras universidades, de forma genérica.

Para pesquisas futuras, sugerem-se 1) a construção de um modelo de avaliação de desempenho para os demais processos-chave (ensino, extensão e gestão financeira e orçamentária) da UM; 2) a aplicação da metodologia MCDA-C em outras universidades e em outros contextos; e 3) o acompanhamento da implementação das ações recomendadas e do desempenho da atividade de pesquisa da Universidade de Mindelo, em anos subsequentes.

\section{REFERÊNCIAS}

Agyemang, G. \& Broadbent, J. (2015) Management control systems and research management in universities: An empirical and conceptual exploration. Accounting Auditing and Accountability, 28(7), 1018-1046.

Asif, M. \& Searcy, C. (2014) A composite index for measuring performance in higher education institutions. International Journal of Quality \& Reliability Management, 31(9), 983-1001.

Azma, F. (2010) Qualitative Indicators for the evaluation of universities performance. Procedia Social and Behavioral Sciences, 2, 5408-5411.

Beerkens, M. (2013) Facts and fads in academic research management: The effect of management practices on research productivity in Australia. Research Policy, 42, 1679-1693.

Behn, R. D. (2003) Why Measure Performance? Different Purposes Require Different Measures. Public Administration Review, 63(5), 586-606.

Bei, W. \& Dongsheng, L. (2014) The Performance Evaluation of University Scientific Research Project Management Based on the FAHP. Journal of Digital Information Management, 12(1), 18-26.

Bititci, U.; Garengo, P. G.; Dörfler, V.; Nudurupati, Sai. (2012) Performance Measurement: Challenges for Tomorrow. International Journal of Management Reviews, 14, 305-327.

Bititci, U. S.; Turner, T. \& Begemann, C. (2000) Dynamics of performance measurement systems. International Journal of Operations \& Production Management, 20 (5-6), 692-704.

Bourne, M.; Mills, J.; Wilcox, M.; Neely, A. \& Platts, K. (2000) Designing, implementing and updating performance measurement systems. International Journal of Operations and Production Management, 20 (7), 754-771.

Cardoso, A. P. B. (2014). Criação do Sistema Nacional de Avaliação Institucional do Ensino Superior em Cabo Verde. In: X Anped Sul, Florianópolis - SC.

Cardoso, T. L.; Ensslin, S. R.; Ensslin, L.; Ripoll-Feliu, V. M. \& Dutra, A. (2015) Reflexões para avanço na área de Avaliação e Gestão do Desempenho das Universidades: 
uma análise da literatura científica. In: Seminários em Administração (XVIII Semead) São Paulo - SP.

Castro, C. M. (1977). A prática da pesquisa. São Paulo: McGraw-Hill do Brasil.

Cullen, J.; Joyce, J.; Hassall, T. \& Broadbent, M. (2003) Quality in higher education: from monitoring to management. Quality Assurance in Education. 11(1), 5-14.

Dias, L. C. \& Tsoukiàs, A. (2004) On the constructive and other approaches in decision aiding. In (Ed.). C.H. Antunes, J. Figueira, J. Clímaco (eds) Aide multicritére à la décision: Multiple criteria decision aiding CCDRC/INESCC/FEUC. Coimbra, 3-28.

Dutra, A.; Ripoll-Feliu, V. M.; Fillol, A. G.; Ensslin, S. R. \& Ensslin, L. (2015) The construction of knowledge from the scientific literature about the theme seaport performance evaluation. International Journal of Productivity and Performance Management, 64(2), 243269.

Ensslin, L.; Neto, G. M. \& Noronha, S. M. (2001). Apoio à decisão: metodologias para estruturação de problemas e avaliação multicritério de alternativas. Insular.

Ensslin, L.; Dutra, A \& Ensslin, S. R. (2000) MCDA: A construtivist approach to the management of human resources at a governmental agency. International Transaciones in Operational Research- ITORS, l7(1), 79-100.

Ensslin, L.; Ensslin, S. R. \& Pinto, H. M. (2013) Processo de investigação e análise bibliométrica: Avaliação da qualidade dos serviços bancários. RAC - Revista de Administração Contemporânea, 17(3), 325-349.

Ensslin, L.; Giffhorn, E.; Ensslin, S. R.; Petri, S. M. \& Vianna, W. B. (2010) Avaliação do desempenho de empresas terceirizadas com o uso da metodologia multicritério de apoio à decisão- construtivista. Revista Pesquisa Operacional, 30(1), 125-152.

Ensslin, S. R.; Dutra, A; Ensslin, L.; Valmorbida, S. M. I. \& Cardoso, T. L.; (2015) Avaliação multicritério de desempenho de portos marítimos brasileiros: estudo de caso para apoiar à gestão do porto de São Francisco do Sul. In Congresso Internacional de Desempenho Portuário (Cidesport). Florianópolis - SC.

Ensslin, S. R.; Ensslin, L.; Imlau, J. M. \& Chaves, L. C. (2014) Processo de mapeamento das publicações científicas de um tema: portfólio bibliográfico e análise bibliométrica sobre avaliação de desempenho de cooperativas de produção agropecuária. Revista de Economia e Sociologia Rural (Impresso), 52(1), 587-608.

Ensslin, S.R.; Ensslin, L.; Back, F. \& Lacerda, R.T.O. (2013) Improved decision aiding in human resource management a case using constructivist multi-criteria decision aiding. International Journal of Productivity and Performance Management, 62(7), 735-757.

Ensslin, L.; Dezem, V.; Dutra, A.; Ensslin, S. R. \& Somensi, K. (2017) Management support for agricultural enterprises: a case study for a fruit-producing company. International Food and Agribusiness Management Review, 19(4), 1-18.

Franco-Santos, M.; Lucianetti, L. \& Bourne, M. (2012) Contemporary performance measurement systems: A review of their consequences and a framework for research. Management Accounting Research, (23), 79-119.

Ghalayini, A. M. \& Noble, J. S. (1996) The changing basis of performance measurement. International Journal of Operations \& Production Management, 16(8), 63-80.

Revista de Gestão e Secretariado-GeSec, São Paulo, v. 8, n. 2, p 76-96, Mai./Ago. 2017. 
Higgins, J. C. (1989) Performance measurement in universities. European Journal of Operational Research, 38, 358-368.

Korhonen, P.; Tainio, R. \& Wallenius, J. (2001) Value efficiency analysis of academic research. European Journal of Operational Research, 130, 121-132.

Lacerda, R. T. O.; Ensslin, L. \& Ensslin, S. R. (2011) A performance measurement framework in portfolio management: A constructivist case. Management Decision, 49(3-4), 648-668.

Longaray, A. A. \& Ensslin, L. (2015) Use of multi-criteria decision aid to evaluate the performance of trade marketing activities of a brazilian industry. Management and Organizational Studies, 2(1), 15-31.

Luna, E. V. (2015) Key performance indicators (KPI) system in education. Asian Social Science, 11(8), 194-200.

Melnyk, S. A.; Bititci, U.; Platts, K.; Tobias, J. \& Andersen, B.( 2014) Is performance measurement and management fit for the future? Management Accounting Research, (25), 173-186.

Prathap, G. \& Ratnavelu, K. (2015) Research performance evaluation of leading higher education institutions in Malaysia. Current Science, 109(6), 1159-1164.

Richardson, R. J. (1999). Pesquisa Social: métodos e técnicas (3a ed.). São Paulo: Atlas.

Roy, B. (1993) Decision science or decision-aid science? European Journal of Operational Research, 66 (2), 184-203.

Thiel, G. G.; Ensslin, S. R. \& Ensslin, L. (2017) Street lighting management and performance evaluation: opportunities and challenges. Lex Localis - Journal of Local SelfGovernment, 15(2), 303-328.

Valmorbida, S. M. I. \& Ensslin, S. R. (2015) Avaliação de desempenho de rankings universitários: revisão da literatura e diretrizes para futuras investigações. In Anais do Encontro da Anpad (XXXIX Enanpad 2015). Belo Horizonte-MG.

Valmorbida, S. M. I.; Ensslin, S. R. \& Ensslin, L. (2014) Avaliação de desempenho para auxílio na gestão de universidades públicas: análise da literatura para identificação de oportunidades de pesquisas. Contabilidade, Gestão e Governança, 17(3), 4-28.

Valmorbida, S. M. I.; Ensslin, S. R.; Ensslin, L. \& Ripoll-Feliu, V. M. (2015) University management with focus on multicriteria performance evaluation: illustration in the brazilian context. GCG: Journal of Globalization, Competitiveness \& Governability, 9(2), 61-75.

Waiczyk, C. \& Ensslin, S. R. (2013) Avaliação de produção científica de pesquisadores: mapeamento das publicações científicas. Revista Contemporânea de Contabilidade, 10(20), 97-112. 